\title{
ANOMALOUS DISSIPATION IN A STOCHASTICALLY FORCED INFINITE-DIMENSIONAL SYSTEM OF COUPLED OSCILLATORS
}

\author{
JONATHAN C. MATTINGLY, TOUFIC M. SUIDAN, ERIC VANDEN-EIJNDEN
}

\begin{abstract}
We study a system of stochastically forced infinite-dimensional coupled harmonic oscillators. Although this system formally conserves energy and is not explicitly dissipative, we show that it has a nontrivial invariant probability measure. This phenomenon, which has no finite dimensional equivalent, is due to the appearance of some anomalous dissipation mechanism which transports energy to infinity. This prevents the energy from building up locally and allows the system to converge to the invariant measure. The invariant measure is constructed explicitly and some of its properties are analyzed.
\end{abstract}

\section{INTRODUCTION}

Consider the infinite dimensional linear system of coupled stochastic differential equations

$$
\left\{\begin{array}{l}
\dot{a}_{n}(t)=a_{n-1}(t)-a_{n+1}(t)+\delta_{n, 1} \dot{W}(t) \quad n \in \mathbb{N} \\
a_{0}(t)=0
\end{array}\right.
$$

where the forcing, $\dot{W}(t)$, is additive white noise and $\delta_{n, 1}$ denotes the Kronecker delta. Formally, the unforced system appears to conserve energy:

$$
\frac{d}{d t}\left(\frac{1}{2} \sum_{n \in \mathbb{N}} a_{n}^{2}\right)=\sum_{n \in \mathbb{N}} a_{n} \dot{a}_{n}=\sum_{n \in \mathbb{N}} a_{n}\left(a_{n-1}-a_{n+1}\right)=\sum_{n \in \mathbb{N}} a_{n} a_{n-1}-\sum_{n \in \mathbb{N}} a_{n} a_{n+1}=0 .
$$

Of course, this calculation is only formal as the rearrangement of the summations is justified only if the sequences involved are absolutely convergent. Since the matrix which encodes the coupling in (1) is real antisymmetric, any finite even dimensional truncation of this matrix has the same real Jordan canonical form as a system of uncoupled simple harmonic oscillators.

Our goal in this paper is to address the following natural question: What is the long term behavior of (1)? More precisely, does the forced system (1) have an invariant measure or statistical steady state? Finite dimensional truncations might lead one to conjecture a negative answer to this question. This is due to the fact that, in the finite dimensional setting, there is no mechanism for energy dissipation. The infinite dimensional system, on the other hand, does indeed give rise to a nontrivial invariant probability measure which is not supported in $\ell_{2}$. Since there is no apparent dissipative mechanism, we loosely refer to this phenomena as anomalous dissipation. The present authors have introduced a formal version of this notion in [3] and have used it to analyze a variety of models.

The invariant probability measure described above is explicitly computable and is given by the formula:

$$
a_{n}(t)=\sqrt{\frac{2}{\pi}} \int_{-\infty}^{t} G_{n}^{0}(t-s) d W(s)
$$

where

$$
G_{n}^{0}(s)=\int_{-1}^{1} \overline{\mathcal{U}_{n-1}(z)} \sqrt{1-z^{2}} e^{2 i z s} d z,
$$

$\mathcal{U}_{n}(z)=i^{n} U_{n}(z)$, and $U_{n}(z)$ is the $n^{\text {th }}$ normalized Chebychev Polynomial of the second kind [1, 4].

In this paper we show that (1) does have a nontrivial invariant probability measure given by (3). We derive the explicit representation for this measure and compute its covariance structure. We also describe a natural class of initial data whose long term dynamics converge to (3). The analysis is technically elementary and involves only classical orthogonal polynomials and basic facts from stochastic calculus. The model analyzed here should be compared to the related models presented in [3] whose structures are similar except that the coefficients of the $a_{n-1}$ and $a_{n+1}$ terms depend on $n$. Those models also exhibit anomalous dissipations, but the qualitative features of their invariant measures 
differ from those of (3) (in particular, the models in [3] have a different covariance structure than (37). The analysis performed in [3], which is based on generating functions, is also different from the one for (3) presented below.

\section{INVARIANT MEASURE}

We first analyze a damped version of (1) in the interest of guessing the inviscid invariant measure (3). Next, we prove that the damped invariant measure converges to the inviscid invariant measure (3). Consider the damped stochastically forced infinite dimensional system:

$$
\left\{\begin{array}{l}
\dot{a}_{n}(t)=a_{n-1}(t)-a_{n+1}(t)-\nu a_{n}(t)+\delta_{n, 1} \dot{W}(t) \\
a_{0}(t)=0,
\end{array}\right.
$$

where $\nu \geq 0$ is a parameter. We show that this equation has a stationary solution given by

$$
a_{n}^{\nu}(t)=\sqrt{\frac{2}{\pi}} \int_{-\infty}^{t} G_{n}^{\nu}(t-s) d W(s)
$$

where

$$
G_{n}^{\nu}(s)=\int_{-1}^{1} \overline{\mathcal{U}_{n-1}(z)} \sqrt{1-z^{2}} e^{-(\nu-2 i z) s} d z .
$$

Before establishing that (5) solves (4), we show that (5) is a well defined random variable. It is sufficient to prove that $\int_{0}^{\infty}\left|G_{n}^{\nu}(s)\right|^{2} d s<\infty$. This is clear for the case $\nu>0$. We present a short argument for the case $\nu=0$. Let $f_{n}^{\gamma}(z)=\mathcal{U}_{n}(z) \sqrt{1-z^{2}} e^{2 i \gamma z}$ where $\gamma \in[0, \pi]$. Note that $\int_{-1}^{1}\left|f_{n}^{\gamma}(z)\right|^{2} d z<\infty$ and independent of $\gamma$. Also note that $G_{n}^{0}(\gamma+k \pi)=\int_{-1}^{1} f_{n-1}^{\gamma}(z) e^{2 \pi i k z} d z$ is the $-k^{t h}$ Fourier coefficient of $f_{n-1}^{\gamma}$. The Plancharel theorem and compactness of $[0, \pi]$ imply that

$$
\sum_{k \in \mathbb{Z}}\left|G_{n}^{0}(\gamma+k \pi)\right|^{2}<\mathcal{C}<\infty
$$

for some constant $\mathcal{C}$ which is independent of $\gamma$. These observations give the desired estimate

$$
\begin{aligned}
\int_{0}^{\infty}\left[G_{n}^{0}(s)\right]^{2} d s & \leq \sum_{k \in \mathbb{Z}} \int_{0}^{\pi}\left|G_{n}^{0}(\gamma+k \pi)\right|^{2} d \gamma=\int_{0}^{\pi}\left[\sum_{k \in \mathbb{Z}}\left|G_{n}^{0}(\gamma+k \pi)\right|^{2}\right] d \gamma \\
& =\int_{0}^{\pi}\left[\int_{-1}^{1}\left|f_{n-1}^{\gamma}(z)\right|^{2} d z\right] d \gamma=\pi\left\|f_{n-1}^{\gamma}\right\|_{2}^{2} \leq \mathcal{C} \pi<\infty
\end{aligned}
$$

and show that (5) is a well defined random variable. In fact, equation (5) defines an infinite dimensional Gaussian Markov process, $\left\{a_{n}^{\nu}(t)\right\}$. The measure that (5) induces is clearly invariant under time shifts of the driving Brownian motion. Thus, once we show that (5) is a solution of equation (4) for all $\nu \geq 0$ the previous observation implies that (5) induces an invariant measure for (4) for all $\nu \geq 0$. Since the invariant Gaussian measure we constructed is explicit, we can calculate its covariance structure: This will be done in section 3

To show that (5) solves (4) for all $\nu \geq 0$, we use the following basic fact about Wiener integrals: For nice $G$, e.g. $G \in C^{1}(\mathbb{R}) \cap H_{1}\left(\mathbb{R}_{+}\right), g(t)=\int_{-\infty}^{t} G(t-s) d W(s)$ satisfies

$$
d g(t)=G(0) d W(t)+\left[\int_{-\infty}^{t} G^{\prime}(t-s) d W(s)\right] d t .
$$

Denote by $\mu(d z)$ the measure $\sqrt{1-z^{2}} d z . G_{1}^{\nu}(0)=\sqrt{\frac{\pi}{2}}$ and the orthogonality of the $\left\{\mathcal{U}_{n}\right\}$ in $L^{2}([-1,1], \mu)$ imply that $G_{n}^{\nu}(0)=0$ for all $n>1$. Calculating the $G_{n}^{\nu \prime}(t-s)$ and using the recurrence relation $(8)$, we arrive at the relation

(7) $G_{n}^{\nu \prime}(t-s)=-\int_{-1}^{1}(\nu-2 i z) \overline{\mathcal{U}_{n-1}(z)} \sqrt{1-z^{2}} e^{-(\nu-2 i z)(t-s)} d z=G_{n-1}^{\nu}(t-s)-G_{n+1}^{\nu}(t-s)-\nu G_{n}^{\nu}(t-s)$.

We recover the equations in (4) by applying (6) and (7) to formula (5) for the sequence $\left\{a_{n}^{\nu}\right\}$ and using the recurrence relation

$$
\mathcal{U}_{n+1}(z)-\mathcal{U}_{n-1}(z)=2 i z \mathcal{U}_{n}(z)
$$


Relation (8) follows directly from the well-known three term recurrence relation for the Chebychev polynomials:

$$
U_{n+1}(z)+U_{n-1}(z)=2 z U_{n} .
$$

This shows that (5) does indeed provide a solution and stationary measure for the infinite dimensional coupled system (4).

Finally, we explain the origin of formula (5) for the $\left\{a_{n}^{\nu}\right\}$. Notice that the coupling matrix of equation (1D) is the Jacobi matrix associated to the three term recurrence of the Chebychev polynomials of the second kind [2, 4]. Therefore, working with generating functions in the Chebychev polynomials will diagonalize (1). With this in mind, consider

$$
\alpha^{\nu}(z, t)=\sum_{n=1}^{\infty} a_{n}^{\nu}(t) \mathcal{U}_{n-1}(z)
$$

Using the recurrence relation (8) and the facts $\mathcal{U}_{0}(z)=\sqrt{\frac{2}{\pi}}$ and $\mathcal{U}_{1}(z)=2 i z \mathcal{U}_{0}(z)$, one can calculate the time derivative of $\alpha^{\nu}(z, t)$ as follows:

$$
\begin{aligned}
\dot{\alpha}^{\nu}(z, t) & =\sum_{n=1}^{\infty} \dot{a}_{n}^{\nu}(t) \mathcal{U}_{n-1}(z) \\
& =\sum_{n=1}^{\infty}\left[a_{n-1}^{\nu}(t)-a_{n+1}^{\nu}(t)-\nu a_{n}^{\nu}(t)+\delta_{n, 1} \dot{W}(t)\right] \mathcal{U}_{n-1}(z) \\
& =-\nu \alpha^{\nu}(z, t)+\sum_{n=1}^{\infty} a_{n-1}^{\nu}(t) \mathcal{U}_{n-1}(z)-\sum_{n=1}^{\infty} a_{n+1}^{\nu}(t) \mathcal{U}_{n-1}(z)+\mathcal{U}_{0}(z) \dot{W}(t) \\
& =-\nu \alpha^{\nu}(z, t)+a_{1}^{\nu}(t) \mathcal{U}_{1}(z)-2 i z a_{1}^{\nu}(t) \mathcal{U}_{0}(z)+2 i z \alpha^{\nu}(z, t)+\mathcal{U}_{0}(z) \dot{W}(t) \\
& =-\nu \alpha^{\nu}(z, t)+2 i z \alpha^{\nu}(z, t)+\mathcal{U}_{0}(z) \dot{W}(t) .
\end{aligned}
$$

This implies that $\alpha^{\nu}(z, t)$ satisfies the stochastic differential equation

$$
\dot{\alpha}^{\nu}(z, t)=-(\nu+2 i z) \alpha^{\nu}(z, t)+\sqrt{\frac{2}{\pi}} \dot{W}(t)
$$

with $z$ viewed as a parameter varying in $[-1,1]$. The solution to the initial value problem for (9) and $t \geq s$ is

$$
\alpha^{\nu}(z, t)=\varphi^{\nu}(t-s, z) \alpha^{\nu}(z, s)+\sqrt{\frac{2}{\pi}} \int_{s}^{t} \varphi^{\nu}(t-r, z) d W(r)
$$

where $\varphi^{\nu}(t, z)=\exp ((2 i z-\nu) t)$. Letting $s \rightarrow-\infty$ and assuming "nice initial conditions" and $\nu>0$, we obtain the form of the invariant measure in formula (5). We note that although this is the way in which the expressions above were derived or "guessed", none of the results depend on this derivation.

\section{Covariance Structure of the Invariant Measure}

We compute the covariance structure of (3), $c^{0}(m, n)=\mathbb{E} a_{m}^{0} a_{n}^{0}$, by first computing the covariance structure of (5), $c^{\nu}(m, n)=\mathbb{E} a_{m}^{\nu} a_{n}^{\nu}$, and taking the limit as $\nu \rightarrow 0$. The justification of this procedure requires two short steps: First, show that there is a sequence of $\nu_{k} \rightarrow 0$ such that $a_{n}^{\nu_{k}}(0) \rightarrow a_{n}^{0}(0)$ almost surely as $k \rightarrow \infty$; second, show that $a_{n}^{\nu}$ is almost surely a uniformly continuous function of $\nu \in(0, M)$ for any $M>0$. Therefore, the convergence of $a_{n}^{\nu} \rightarrow a_{n}^{0}$ as $\nu \rightarrow 0$ holds almost surely. The following estimate and the Borel-Cantelli lemma complete the first step:

$$
\mathbb{P}\left(\left|a_{n}^{\nu_{k}}(0)-a_{n}^{0}(0)\right|>\frac{1}{k}\right) \leq k^{2} \int_{0}^{\infty}\left|e^{-\nu_{k} t}-1\right|^{2}\left|G_{n}^{0}(t)\right|^{2} d t .
$$

To apply the Borel-Cantelli lemma, simply choose $\nu_{k}$ so that the sum over $k$ of the right hand side of (11) is finite. For such a choice of $\nu_{k}, a_{n}^{\nu_{k}}(0) \rightarrow a_{n}^{0}(0)$ almost surely. To show that $a_{n}^{\nu}(0)$ is almost surely a uniformly continuous 
function of $\nu \in(0, M)$, we appeal to the Kolmogorov continuity theorem and the following estimate. Fix $0<\eta<\frac{1}{2}$, $\rho>\nu>0$, and observe that

$$
\begin{aligned}
\mathbb{E}\left|a_{n}^{\nu}(0)-a_{n}^{\rho}(0)\right|^{2} & =\int_{0}^{\infty} e^{-2 \nu t}\left|1-e^{-(\rho-\nu) t}\right|^{2}\left|G_{n}^{0}(t)\right|^{2} d t \\
& =\int_{0}^{|\rho-\nu|^{-\eta}} e^{-2 \nu t}\left|1-e^{-(\rho-\nu) t}\right|^{2}\left|G_{n}^{0}(t)\right|^{2} d t+\int_{|\rho-\nu|^{-\eta}}^{\infty} e^{-2 \nu t}\left|1-e^{-(\rho-\nu) t}\right|^{2}\left|G_{n}^{0}(t)\right|^{2} d t \\
& \leq C_{1}|\rho-\nu|^{2(1-\eta)}+C_{2} e^{-2 \nu|\rho-\nu|^{-\eta}} \leq C_{3}|\rho-\nu|^{2(1-\eta)},
\end{aligned}
$$

where the last inequality holds for $|\rho-\nu|$ sufficiently small and $C_{1}, C_{2}$, and $C_{3}$ are constants which do not depend on $\eta, \nu$, and $\rho$. This completes the justification for computing $c^{0}(m, n)$ by taking the limit of $c^{\nu}(m, n)$ as $\nu \rightarrow 0$.

We now compute $c^{\nu}(n, n)$ :

$$
\begin{aligned}
\mathbb{E} a_{n}^{\nu}(0) \overline{a_{n}^{\nu}(0)} & =\frac{2}{\pi} \int_{0}^{\infty} G_{n}^{\nu}(s) \overline{G_{n}^{\nu}(s)} d s \\
& =\frac{2}{\pi} \int_{0}^{\infty}\left[\int_{-1}^{1} \overline{\mathcal{U}_{n-1}(z)} \sqrt{1-z^{2}} e^{-(\nu-2 i z) s} d z\right] \overline{\left[\int_{-1}^{1} \overline{\mathcal{U}_{n-1}\left(z^{\prime}\right)} \sqrt{1-z^{\prime 2}} e^{-\left(\nu-2 i z^{\prime}\right) s} d z^{\prime}\right]} d s \\
& =\frac{2}{\pi} \int_{-1}^{1} \int_{-1}^{1} \overline{\mathcal{U}_{n-1}(z)} \mathcal{U}_{n-1}\left(z^{\prime}\right) \sqrt{1-z^{2}} \sqrt{1-z^{\prime 2}}\left[\int_{0}^{\infty} e^{-2\left(\nu-i\left(z-z^{\prime}\right)\right) s} d s\right] d z d z^{\prime} \\
& =\frac{2}{\pi} \int_{-1}^{1} \int_{-1}^{1} U_{n-1}(z) U_{n-1}\left(z^{\prime}\right) \frac{\sqrt{1-z^{2}} \sqrt{1-z^{\prime 2}}}{2 \nu-2 i\left(z-z^{\prime}\right)} d z d z^{\prime} .
\end{aligned}
$$

The fact that the dynamics of $\left\{a_{n}\right\}$ is real implies that one needs only to compute the real part of equation (12):

$$
\frac{\nu}{\pi} \int_{-1}^{1} \int_{-1}^{1} U_{n-1}(z) U_{n-1}\left(z^{\prime}\right) \frac{\sqrt{1-z^{2}} \sqrt{1-z^{\prime 2}}}{\nu^{2}+\left(z-z^{\prime}\right)^{2}} d z d z^{\prime}
$$

Introducing the change of variables $z=\cos (\pi \theta), z^{\prime}=\cos \left(\pi \theta^{\prime}\right)$ and using the fact that $U_{n}(\cos (\pi \theta))=\sqrt{\frac{2}{\pi}} \frac{\sin (\pi(n+1) \theta)}{\sin (\pi \theta)}$, see for example [4], leads to the following integral:

$$
c^{\nu}(n, n)=2 \nu \int_{0}^{1} \int_{0}^{1} \frac{\sin (\pi n z) \sin \left(\pi n z^{\prime}\right) \sin (\pi z) \sin \left(\pi z^{\prime}\right)}{\nu^{2}+\left(\cos (\pi z)-\cos \left(\pi z^{\prime}\right)\right)^{2}} d z d z^{\prime}
$$

Analyzing the limit $\nu \rightarrow 0$, one finds that:

$$
c^{0}(n, n)=\lim _{\nu \rightarrow 0} c^{\nu}(n, n)=\int_{0}^{2} \sin \left(\pi \frac{\xi}{2}\right) \sin ^{2}\left(\pi \frac{n \xi}{2}\right) d \xi .
$$

Note that $\lim _{n \rightarrow \infty} c^{0}(n, n)=\frac{2}{\pi}$ which immediately shows that the invariant measure is not supported on $\ell_{2}$ : We will discuss the implication of this fact in Section 4 For the moment, we only remark that the existence of this limit is consistent with the systems' invariance by translation except for the forcing and the boundary condition at $n=0$.

We compute the general covariance structure $c^{\nu}(m, n)$ in two steps: $n-m$ is odd; $n-m$ is even. First note that

$$
\mathbb{E} a_{n}^{\nu}(0) \overline{a_{m}^{\nu}(0)}=\frac{(-1)^{n}}{\pi} i^{n+m} \int_{-1}^{1} \int_{-1}^{1} U_{n-1}(z) U_{m-1}\left(z^{\prime}\right) \sqrt{1-z^{2}} \sqrt{1-\left(z^{\prime}\right)^{2}} \frac{\nu+i\left(z-z^{\prime}\right)}{\nu^{2}+\left(z-z^{\prime}\right)^{2}} d z d z^{\prime} .
$$

The cases when $n-m$ is odd or even must be treated separately:

Case 1: If $n-m$ is odd, then

$$
\begin{aligned}
& \mathbb{E} a_{n}^{\nu}(0) \overline{a_{m}^{\nu}(0)}=\frac{(-1)^{n}}{\pi} i^{n+m+1} \int_{-1}^{1} \int_{-1}^{1} U_{n-1}(z) U_{m-1}\left(z^{\prime}\right) \sqrt{1-z^{2}} \sqrt{1-\left(z^{\prime}\right)^{2}} \frac{\left(z-z^{\prime}\right)}{\nu^{2}+\left(z-z^{\prime}\right)^{2}} d z d z^{\prime} \\
& \mathbb{E} a_{m}^{\nu}(0) \overline{a_{n}^{\nu}(0)}=\frac{(-1)^{m}}{\pi} i^{n+m+1} \int_{-1}^{1} \int_{-1}^{1} U_{n-1}(z) U_{m-1}\left(z^{\prime}\right) \sqrt{1-z^{2}} \sqrt{1-\left(z^{\prime}\right)^{2}} \frac{\left(z-z^{\prime}\right)}{\nu^{2}+\left(z-z^{\prime}\right)^{2}} d z d z^{\prime} .
\end{aligned}
$$


Since $n-m$ is odd, these two expressions have opposite signs. On the other hand, the fact that the dynamics of the $\left\{a_{n}\right\}$ is real implies that the two expressions must be equal. Therefore, $c^{\nu}(m, n)=0$ which further implies that $c^{0}(m, n)=0$.

Case 2: If $n-m$ is even, then

$$
\mathbb{E} a_{n}^{\nu}(0) \overline{a_{m}^{\nu}(0)}=\frac{(-1)^{n}}{\pi} i^{n+m} \nu \int_{-1}^{1} \int_{-1}^{1} U_{n-1}(z) U_{m-1}\left(z^{\prime}\right) \sqrt{1-z^{2}} \sqrt{1-\left(z^{\prime}\right)^{2}} \frac{1}{\nu^{2}+\left(z-z^{\prime}\right)^{2}} d z d z^{\prime} .
$$

Once again, analyzing the limit of (17) as $\nu \rightarrow 0$ leads to the formula for $c^{0}(m, n)$ :

$$
\begin{aligned}
c^{0}(m, n)=\lim _{\nu \rightarrow 0} c^{\nu}(m, n) & =(-1)^{n} i^{m+n} \int_{0}^{2} \sin \left(\pi \frac{\xi}{2}\right) \sin \left(\pi \frac{m \xi}{2}\right) \sin \left(\pi \frac{n \xi}{2}\right) d \xi \\
& =(-1)^{n} i^{n+m} \frac{2}{\pi}\left[\frac{1}{(n+m)^{2}-1}-\frac{1}{(n-m)^{2}-1}\right]
\end{aligned}
$$

\section{Basin of Attraction of the InVariant Measure}

Next, we prove that if the initial condition, $\left\{a_{n}\right\}$, is in $\ell_{2}$, i.e. $\sum_{n=1}^{\infty}\left|a_{n}\right|^{2}<\infty$, then the dynamics converges weakly to the invariant measure (5) for any $\nu \geq 0$ : Any finite collection of coordinates, $a_{i_{1}}^{\nu}, \ldots, a_{i_{k}}^{\nu}$, converges to (5) as the initial condition is pulled back to $s=-\infty$. Assume that $\left\{a_{n}\right\} \in \ell_{2}$ and construct the function

$$
\alpha_{0}^{\nu}(z)=\sum_{n=1}^{\infty} a_{n} \mathcal{U}_{n-1}(z)
$$

If $\alpha^{\nu}(z, s)=\alpha_{0}^{\nu}(z)$ is the initial condition at time $s$ for equation (9), then the solution at time $t>s$ is given by equation (10). Note that the dynamics is well defined for $\ell_{2}$ initial data and the solution remains in $\ell_{2}$ for all $t<\infty$. To recover the solution at time $t$ we simply use orthogonality of the $\left\{\mathcal{U}_{n}\right\}$ :

$$
\begin{aligned}
a_{n}^{\nu}(t)= & \int_{-1}^{1} \overline{\mathcal{U}_{n-1}(z)} \alpha^{\nu}(z, t) \sqrt{1-z^{2}} d z \\
= & \int_{-1}^{1} \overline{\mathcal{U}_{n-1}(z)} \varphi^{\nu}(t-s, z) \alpha^{\nu}(z, s) \sqrt{1-z^{2}} d z \\
& +\int_{-1}^{1} \overline{\mathcal{U}_{n-1}(z)}\left[\sqrt{\frac{2}{\pi}} \int_{s}^{t} \varphi^{\nu}(t-r, z) d W(r)\right] \sqrt{1-z^{2}} d z .
\end{aligned}
$$

Since $\overline{\mathcal{U}_{n-1}(z)} \alpha_{0}^{\nu}(z) \sqrt{1-z^{2}} \chi_{[-1,1]}(z) \in L^{1}(\mathbb{R})$, standard Fourier analysis implies that the first integral vanishes as $s \rightarrow-\infty$. The second integral converges to the form of the invariant measure (5). The convergence is uniform if a finite collection of coordinates is fixed. Therefore, for any bounded cylinder function, convergence is established, which, in turn, establishes weak convergence of solutions with $\ell_{2}$ initial data to the invariant measure (5).

A drawback of the convergence result above is that, when $\nu=0$, the invariant measure is not supported on $\ell_{2}$. Hence, we may wonder about convergence of initial data which is not in $\ell_{2}$ and is in the support of the invariant measure or even in $\ell_{\infty}$. This question, however, turns out to be quite complicated as the behavior of the initial value problem for (1) depends sensitively on the initial condition. Since (1) is linear, it suffices to understand the solution of the unforced system with initial condition $a_{n}(s)=a_{n}^{0}$ :

$$
\left\{\begin{array}{l}
\dot{a}_{n}(t)=a_{n-1}(t)-a_{n+1}(t), \quad a_{n}(0)=a_{n}^{0} \quad n \in \mathbb{N} \\
a_{0}(t)=0 .
\end{array}\right.
$$

The solution of the forced system with initial condition $a_{n}(s)=a_{n}^{0}$ is then obtained by adding the solution of (1) with $a_{n}(s)=0$ to that of (21). Cataloging the behavior of the solutions of (21) is complicated, even if we restrict ourselves to initial condition in $\ell_{\infty}$. The difficulty is immediately understandable if one notices that

$$
a_{2 n}=1, \quad a_{2 n+1}=0, \quad n \in \mathbb{N}
$$

is a fixed point for (21) and belongs to $\ell_{\infty}$. Similarly, one can find time-periodic solutions with arbitrary period $T>0$ which also belong to $\ell_{\infty}$. For the sake of brevity, we will refrain from attempting a complete analysis of (21). We note, however, that such an analysis has been performed in detail in [3] for models related to [21]. 
Acknowledgments: We thank Percy Deift, Charles Fefferman, Stephanos Venakides, and Xin Zhou for useful conservations. J. Mattingly is supported in part by the Sloan Foundation and by an NSF CAREER award DMS04-49910. T. Suidan is supported in part by NSF grant DMS05-53403. E. Vanden-Eijnden is supported in part by NSF grants DMS02-09959 and DMS02-39625, and by ONR grant N00014-04-1-0565.

\section{REFERENCES}

[1] M. Abramowitz and I. Stegun, editors. Handbook of mathematical functions with formulas, graphs, and mathematical tables. Dover Publications Inc., New York, 1992. Reprint of the 1972 edition.

[2] P. A. Deift. Orthogonal polynomials and random matrices: a Riemann-Hilbert approach, volume 3 of Courant Lecture Notes in Mathematics. New York University Courant Institute of Mathematical Sciences, New York, 1999.

[3] J. C. Mattingly, T. M. Suidan, and E. Vanden-Eijnden. Simple Systems with Anomalous Dissipation and Energy Cascade. arxiv.org/abs/mathph/0607047.

[4] G. Szegö. Orthogonal Polynomials, volume 23 of American Mathematical Society, Colloquium Publications. AMS, Providence, R.I., fourth edition, 1975. 